\title{
Téoros
}

Revue de recherche en tourisme

\section{Aspects sociogéographiques du développement du tourisme balnéaire au Maroc}

\section{Mimoun Hillali}

Volume 24, numéro 1, printemps 2005

Maroc, réalités et défis

URI : https://id.erudit.org/iderudit/1071103ar

DOI : https://doi.org/10.7202/1071103ar

Aller au sommaire du numéro

Éditeur(s)

Université du Québec à Montréal

ISSN

0712-8657 (imprimé)

1923-2705 (numérique)

Découvrir la revue

Citer cet article

Hillali, M. (2005). Aspects sociogéographiques du développement du tourisme balnéaire au Maroc. Téoros, 24(1), 6-11. https://doi.org/10.7202/1071103ar d'utilisation que vous pouvez consulter en ligne.

https://apropos.erudit.org/fr/usagers/politique-dutilisation/ 


\section{Aspects sociogéographiques du développement du tourisme balnéaire au Maroc}

\section{Mimoun Hillali}

Séduit par le succès du tourisme sur la rive nord de la Méditerranée (décennie 1960), le Maroc cède à la tentation de jumeler le tourisme et l'agriculture pour en faire les leviers de son développement social et économique. N'étant pas un pays producteur de pétrole, encouragé par les recommandations de l'expertise internationale et jouissant d'une bonne image auprès des pays occidentaux, principaux foyers émetteurs de touristes, le choix qu'il fait du tourisme paraît découler de réflexions mûrement pensées.

Cependant, replacée dans le contexte sociopolitique du Maroc indépendant et du climat tendu de la guerre froide, cette décision s'explique également par des considérations nationales et des pressions internationales évidentes. Cela dit, quoique pertinent, ce choix a provoqué des inquiétudes en son temps quant à sa mise en œuvre.

Hissé au second rang des priorités économiques nationales (après l'agriculture), le tourisme a certes des atouts incontestables, mais à l'état brut (nature, culture, patrimoine...), et ne dispose pas des infrastructures de base ni des moyens d'accompagnement qui en font, sous d'autres cieux, une «industrie » rentable. Autrement dit, tout reste à faire en termes de financement, d'aménagement et de fonctionnement.

Cette importance soudaine accordée au tourisme a mis en évidence ses carences héritées de la période du protectorat : les quelques stations touristiques réalisées et gérées, presque exclusivement, par les colons n'ont généré ni savoir-faire touristique ni avantages économiques en faveur des nationaux. Fort heureusement, une belle plage de sable fin inondée de soleil suffit à faire le bonheur du va-

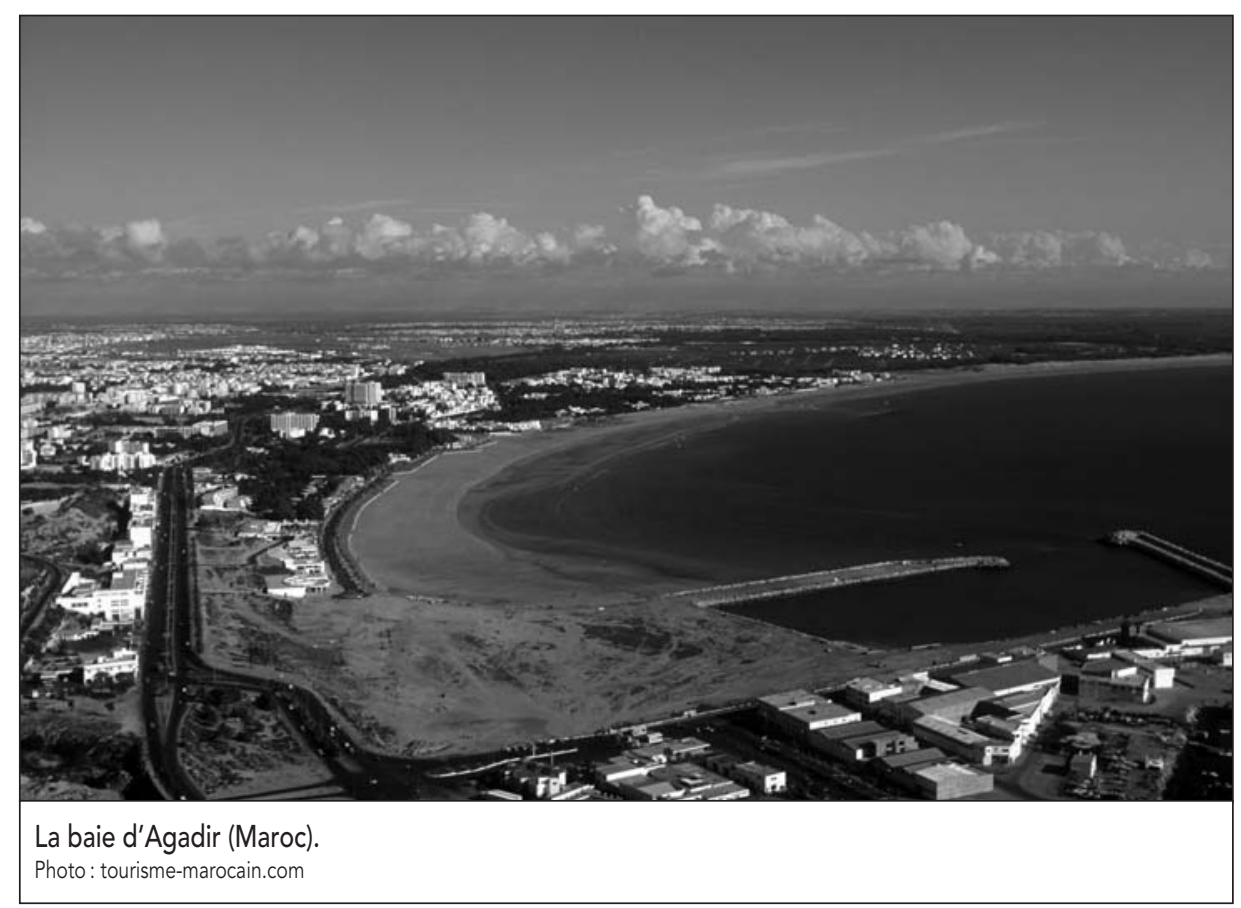

cancier des années 1960. C'est un argument de taille en faveur du tourisme balnéaire. Alors les planificateurs marocains, conseillés par des experts internationaux ${ }^{1}$, se penchent sur les atouts et les contraintes socioéconomiques du pays et étudient notamment :

- La place à réserver au tourisme au sein des autres vocations géographiques et économiques, en veillant à ne pas heurter la sensibilité d'une forte opposition marxisante ou nationaliste, adepte de "l'industrie industrialisante ", voire du collectivisme autogéré ${ }^{2}$.

- Le type de tourisme à privilégier (de masse ou de luxe ?) pour garantir la réussite d'une stratégie " développante » et à éviter, en cas de flottement, de déboucher sur une épreuve de force entre partisans et adversaires de cette nouvelle option.
- Les sources de financement internes et externes, à solliciter, et dans quelles proportions, afin de disposer des capitaux nécessaires à l'aménagement, à l'équipement et à la promotion de stations qu'il faudra créer de toutes pièces.

- Les régions à privilégier dans un premier temps pour séduire, sans paraître reconduire la politique du protectorat, autrefois basée sur la triple notion d'utilité, de rentabilité et de sécurité en faveur du capital colonial.

- Le type d'activité touristique à privilégier dans les régions sélectionnées en prenant en considération l'importance qualitative ou quantitative des autres vocations.

- La définition des moyens juridiques, socioéconomiques et politiques à mettre progressivement en place pour soutenir le secteur dans sa mission de «moteur de développement ». 
En passant en revue ces points clés, indispensables à l'élaboration du plan triennal 1965-1967, les spécialistes de l'époque ont décidé de privilégier le tourisme de manière générale et le tourisme balnéaire de façon particulière. Inopportunément, les agitations sociopolitiques nationales et les crises économiques internationales de la décennie 1970 ont vite fait de dévier la stratégie arrêtée de sa trajectoire initiale.

Près de quarante ans plus tard, le gouvernement marocain remet à I'honneur la même option (tourisme balnéaire) en décidant de créer six nouvelles stations balnéaires de grande envergure, dont une seule cette fois-ci est localisée sur le littoral méditerranéen. Tentative d'équilibrage ou accentuation des inégalités régionales? Les avis divergent à ce sujet, comme par le passé.

\section{Bref aperçu sur les débuts du tourisme au Maroc: Les raisons d'un développement non maîtrisé}

À la signature du traité du protectorat (1912), les autorités coloniales ont compris tout l'intérêt qu'il y avait à mettre conjointement en exergue la richesse et l'exotisme du pays pour attirer l'investisseur et le touriste. De plus, l'incitation des métropolitains à faire le " voyage marocain " a ouvert toutes grandes les occasions d'affaires, souvent grâce à un premier contact. L'espoir d'une ruée vers l'Afrique du Nord et vers l'Afrique tout court a aiguisé l'appétit de certains Européens en mal de fortune, qui rêvaient de reproduire l'épopée américaine en terre africaine.

\section{Le tourisme balnéaire au temps du protectorat: première génération de stations touristiques}

Les prémices du tourisme moderne sont apparues à l'époque du protectorat français et espagnol (1912-1956), dans un Maroc jusqu'ici ${ }^{3}$ familiarisé avec les seuls loisirs traditionnels ${ }^{4}$. Toutefois, avant cette date, les grandes villes avaient développé - depuis des siècles - ce qui est apparemment l'ancêtre de l'hôtel d'aujourd'hui : le Fondouk ${ }^{5}$, sorte de caravansérail urbain.

Un rappel succinct des grandes lignes du tourisme durant la période coloniale permet d'en comprendre l'évolution, ainsi que le dualisme « tourisme culturel et tourisme balnéaire » - un dualisme à vrai dire complé- mentaire dans le temps et dans l'espace -, mais aussi les trébuchements. Les premières initiatives touristiques ${ }^{6}$ remontent aux années 1920. Elles seront perturbées par la guerre du Rif (1921-1926), par la Seconde Guerre mondiale (1940-1945) et par le déclenchement de la lutte pour la libération (1949-1955).

À la veille de l'indépendance, un réseau de petites stations balnéaires, satellites des villes où résident en grand nombre les colons, s'égrènent de Saidia au nord-est à Agadir au sud-ouest. Moulay Bousselham, Mahdia et Oualidia surgissent sur la côte atlantique comme destinations touristiques estivales des citadins et des fermiers des plaines agricoles du Gharb, du Saïs et des Doukkala, fiefs agricoles de la colonisation. Les petites plages périurbaines ou satellites de Rabat, de Casablanca et de Mohammedia attirent les Européens de cet axe économique et administratif.

Au sud, Agadir, aujourd'hui le joyau du tourisme balnéaire du Maroc, n'a pas percé, malgré le titre flatteur de " Nice marocaine » que les Français lui ont attribué. Vers 1959 (Péré, 1972: 7), la ville comptait quelque 500 lits, répartis en 200 chambres de luxe et 60 chambres de catégorie moyenne. À l'intérieur des terres, d'autres stations jalonnent les versants nord du Moyen et du Haut Atlas (Immouzzer, Ifrane, Azrou, Oukaimeden...) et captent les flux touristiques en provenance

\section{Tableau 1}

Programmes et plans de développement socioéconomiques au Maroc, 1949-1992*

\begin{tabular}{|c|c|c|c|}
\hline \multicolumn{2}{|c|}{ Programmes et plans de développement de 1949 à 1992} & \multirow{2}{*}{$\begin{array}{c}\text { Part du budget total (\%) } \\
\qquad 1,24 \%\end{array}$} & \multirow{2}{*}{$\begin{array}{c}\text { Charges du secteur privé } \\
\qquad 93,0 \%\end{array}$} \\
\hline Programme d'équipement & $1949-1952$ & & \\
\hline Programme d'équipement & $1954-1957$ & $0,23 \%$ & - \\
\hline Programme d'équipement & $1958-1959$ & $22,0 \%$ & - \\
\hline Plan quinquennal & $1960-1964$ & $1,40 \%$ & $92,0 \%$ \\
\hline Plan triennal & $1965-1967$ & $6,40 \%$ & $20,0 \%$ \\
\hline Plan quinquennal & $1968-1972$ & $6,80 \%$ & $18,0 \%$ \\
\hline Plan quinquennal & $1973-1977$ & $6,50 \%$ & $16,0 \%$ \\
\hline Plan triennal & $1978-1980$ & $3,40 \%$ & $90,6 \%$ \\
\hline Plan quinquennal & $1981-1985$ & $1,80 \%$ & $90,0 \%$ \\
\hline Plan quinquennal & $1988-1992$ & $1,20 \%$ & $94,0 \%$ \\
\hline Période « blanche »** & $1993-1999$ & - & $96,0 \%$ \\
\hline
\end{tabular}

* En 1992, le Maroc a suspendu la planification pour la reprendre en 1999.

** Estimation personnelle.

Source : Le tourisme international vu du Sud (Mimoun Hillali, 2003 : 43). de Fès, de Meknès ou de Marrakech. Les villes impériales, elles, ont établi et gardé le monopole du tourisme culturel alors que les oasis du Sud ne s'ouvrent au tourisme de découverte qu'au milieu des années 1930, à Sud (cf. bataille de Bougafer, 1934). En somme, le bilan touristique du protectorat est plus que modeste.

\section{L'indépendance et la période de tâtonnement: les raisons économiques d'un choix ou le grand tournant}

En 1956, le Maroc indépendant hérite d'environ 240 hôtels (toutes catégories confondues), d'une capacité de 7300 chambres (15000 lits environ) ; cette capacité demeure presque inchangée de 1956 à 1964, du fait du tâtonnement des gouvernements successifs qui ont, de surcroit, décidé que le tourisme est du ressort du secteur privé (tableau 1).

Cependant, les nouveaux choix économiques du Maroc indépendant se justifient aussi par d'autres difficultés socioéconomiques, comme en attestent quelques chiffres relatifs à l'état de l'héritage colonial global. Selon Salmi (1989: 28), en 1957, de 5 à $10 \%$ des agriculteurs possèdent $60 \%$ de la surface cultivée et la production des paysans et des fellahs n'est commercialisée qu'à hauteur de $30 \%$. Dans les villes, envila fin de la résistance armée dans le Grand 
ron $60 \%$ des 175000 artisans (estimation pour 1960) souffrent du sous-emploi. Quant aux secteurs traditionnels, ils représentent $70 \%$ de la population active. Enfin, le secteur moderne est extrêmement dépendant des capitaux, des débouchés et des experts étrangers. Le pourcentage des travailleurs employés dans les branches secondaires est très réduit : $5 \%$ de la main-d'œuvre totale.

Dans ces conditions, les stations touristiques héritées du protectorat font penser à des ghettos de luxe implantés dans un océan de dénuement. Mais n'est-ce pas là tout le secret de l'attrait des sociétés traditionnelles que protège un enclavement persistant, gardien de mœurs et d'us sans lesquels l'exotisme cesserait de séduire?

Dire qu'un climat ensoleillé constitue un atout touristique important est un lieu commun. Mais affirmer qu'un tel climat est un produit fondamental, cela demande à être vérifié. Jusqu'à une époque récente, seul le « beau temps » était recherché par le touriste acculé à voyager uniquement durant les vacances estivales. Selon Besancenot (1989: 15), "c'est, à l'évidence, le soleil et le 'beau temps' de l'été qui, au lendemain de la Seconde Guerre mondiale, ont fondé la fortune touristique du bassin méditerranéen. C'est la chaleur qui attire de plus en plus dans les zones intertropicales ». Sur ce plan, le Maroc a tout pour plaire (tableau 2).

\section{L'attrait de l'offre originelle: et si le climat était à l'origine de l'adoption du tourisme?}

Mais, aujourd'hui, toutes les composantes du climat sont mises à contribution pour diversifier le produit touristique et permettre l'étalement des vacances. Ainsi, vendre l'inconfort valorisant, y compris le "mauvais temps », est une pratique acquise. La lutte contre les limites de la saisonnalité pour des besoins de rentabilité milite aussi en faveur de la décongestion des régions d'accueil (transport, hébergement, routes...). L'importance donnée au corps (recherche de sensations fortes) a bouleversé la donne sur terre et sur mer : ski, sport nautique, alpinisme, randonnée... L'apparition de ces nouvelles pratiques, souvent animées par des nationaux, ajoutée aux crises économiques et aux violences politiques mondiales, font trébucher le tourisme du tiers-monde et particulièrement sa branche balnéaire au potentiel concentré dans le temps (été) et dans l'espace (littoral).

\section{Le développement du tourisme balnéaire au Maroc, ou l'histoire du verre à moitié vide ou à moitié plein}

N'est-ce pas une décennie d'hésitation improductive (1956-1964) qui a poussé le Maroc à faire du tourisme un secteur prioritaire? Le discours royal ${ }^{7}$ de 1964 est clair à ce propos :

L'accroissement de l'agriculture est une nécessité absolue. C'est par cet accroissement que l'alimentation sera assurée pour les citoyens [...] Notre choix pour le tourisme, comme étant un domaine de priorité, est motivé par le fait que notre pays dispose de possibilités naturelles

\section{Tableau 2}

Moyennes climatiques annuelles des principales stations balnéaires du littoral marocain

\begin{tabular}{l|c|c|c|c|c|c}
\hline Station & $\begin{array}{c}\text { Température } \\
\text { moyenne } \\
\text { annuelle }\end{array}$ & $\begin{array}{c}\text { Pluviométrie } \\
\text { annuelle } \\
\text { (en mm) }\end{array}$ & $\begin{array}{c}\text { Nombre } \\
\text { de jours } \\
\text { de pluie }\end{array}$ & $\begin{array}{c}\text { Température } \\
\text { de l'eau } \\
\text { de mer }\end{array}$ & $\begin{array}{c}\text { Nombre } \\
\text { de jours de } \\
\text { brouillard }\end{array}$ & $\begin{array}{c}\text { Nombre d'heures } \\
\text { d'ensoleillement } \\
\text { par an }\end{array}$ \\
\hline Al Hoceima & $17,5^{\circ}$ & 590 & 69 & $18,1^{\circ}$ & 9 & 2861 \\
Cabo Negro & $15,9^{\circ}$ & 578 & 93 & $17,6^{\circ}$ & - & 2710 \\
Tanger & $17,2^{\circ}$ & 852 & 89 & $17,6^{\circ}$ & 10 & 2987 \\
Rabat & $16,9^{\circ}$ & 518 & 79 & $18,0^{\circ}$ & 41 & 2906 \\
El Jadida & $17,0^{\circ}$ & 375 & 65 & $17,4^{\circ}$ & 27 & 2793 \\
Essaouira & $17,2^{\circ}$ & 279 & 50 & $16,8^{\circ}$ & - & 3009 \\
Agadir & $18,0^{\circ}$ & 240 & 35 & $17,6^{\circ}$ & 34 & 3089 \\
Laâyoune & $20,4^{\circ}$ & 26 & 08 & $18,2^{\circ}$ & - & 3175 \\
\hline
\end{tabular}

Sources: Service météorologique de Casablanca et le Gisement solaire marocain, 1983. qui attirent les touristes désireux de retrouver le repos, le calme et le pittoresque, et animés par l'esprit de savoir et d'apprendre [...]. Le troisième champ d'action auquel nous avons tenu à attribuer la priorité est la formation des cadres [...].

\section{Le tourisme sur le littoral méditerranéen du Maroc: naissance de la deuxième génération de stations balnéaires}

Pour le gouvernement, l'aménagement des stations balnéaires a l'avantage d'engendrer des entités groupées en grappes qui permettent la maîtrise de l'espace et le contrôle des activités, sans oublier l'effet d'entraînement sur les autres régions, contrairement au tourisme itinérant qui nécessite, en plus d'infrastructures suffisantes, des installations dispersées et des moyens d'encadrement conséquents - à l'exception des villes impériales situées dans «l'ex-carré utile » du protectorat. Et, comme par hasard, le Sud intérieur, seule $\mathrm{ZAP}^{8}$ non littorale, ne profite pas de la manne financière des années de l'État prodigue.

Dans la variante tourisme balnéaire, l'État a concentré l'essentiel des projets et des investissements sur le littoral méditerranéen, pensant probablement récupérer les surplus touristiques d'une Espagne qui paraissait, vue de la rive sud, saturée de baigneurs. En consacrant aux opérations d'aménagement touristique et à la construction de structures d'hébergement près des trois quarts des capitaux touristiques publics engagés de 1960 à 1977, les planificateurs et les acteurs touristiques ont opté pour des actions-promotions étatisées volontaristes.

Faute de capitaux privés, le tourisme est confié aux bons soins du secteur public, qui a plutôt veillé à son propre développement. L'importance démesurée accordée aux sociétés étatiques (25\% de la formation brute du capital fixe et $27 \%$ des salaires) a souvent laminé le Trésor (dépenses insensées, déficits chroniques...). Du coup, le processus de liquidation du début de la décennie 1990 est pour l'État une excellente occasion de récupérer des fonds longtemps gelés, de surcroit peu rentables, par le biais de la privatisation, pour combler les déficits en infrastructures légués par une décennie d'ajustement structurel, à un moment où la mondialisation s'affirme au détriment du communisme. En ouvrant le 
tourisme à la privatisation et le balnéaire à de nouvelles activités (plaisance, sport nautique...), le Maroc a pu juguler la crise. La plaisance, pratiquée timidement par les ports d'Asilah, de Tanger et d'Agadir, est rehaussée par les ports modernes de Restinga Smir (quai de plaisance de 2080 mètres linéaires) et de Kabila (498 mètres). Avec l'acheminement vers la métropolisation du bipôle Tanger-Tétouan, grâce à la construction d'un port de renommée internationale sur le Détroit, les stations du Nord ont une chance de revivre.

\section{L'émergence de la station balnéaire d'Agadir: de l'apogée à l'essoufflement !}

Dans le Sud atlantique, la célèbre station balnéaire d'Agadir, prise en charge par le secteur privé, a connu un succès remarquable et dispute la première place, sur l'échiquier touristique national, à Marrakech, la célèbre capitale du tourisme culturel. La station balnéaire du Souss fait partie du « triangle d'or » du tourisme marocain, délimité par les villes d'Agadir, de Marrakech et d'Ouarzazate. Dans ce pôle d'excellence, Agadir s'est imposée en partant de zéro et sans l'interventionnisme étatique constaté ailleurs. De 1948 à 1960, Agadir a pu atteindre une capacité globale supérieure à 1000 lits, avant qu'un violent tremblement de terre ${ }^{9}$ ne réduise la ville à néant. Dix ans plus tard, (1961-1971), elle réalise l'exploit de mettre sur le marché touristique près de 4000 lits, soit $10 \%$ de la capacité d'hébergement nationale (Berriane, 1979: 114). En 1978, Agadir passe en tête des destinations touristiques marocaines avec une capacité d'hébergement de 10230 lits. Elle atteint en 2002 la fabuleuse capacité de 22716 lits sur les 102097 lits classés recensés au Maroc.

L'atout majeur de cette station balnéaire réside dans la qualité de son climat que d'aucuns qualifient d'exceptionnel, mais aussi dans celle de ses acteurs et de ses promoteurs. Contrairement aux stations méditerranéennes (investissements publics et semi-publics considérables mais peu rentables), la baie d'Agadir n'a pas profité de la manne financière étatique des années 1960 et 1970, même si elle s'était dotée d'une Société nationale d'aménagement touristique, la Société nationale d'aménagement de la baie d'Agadir (Sonaba) ${ }^{10}$.

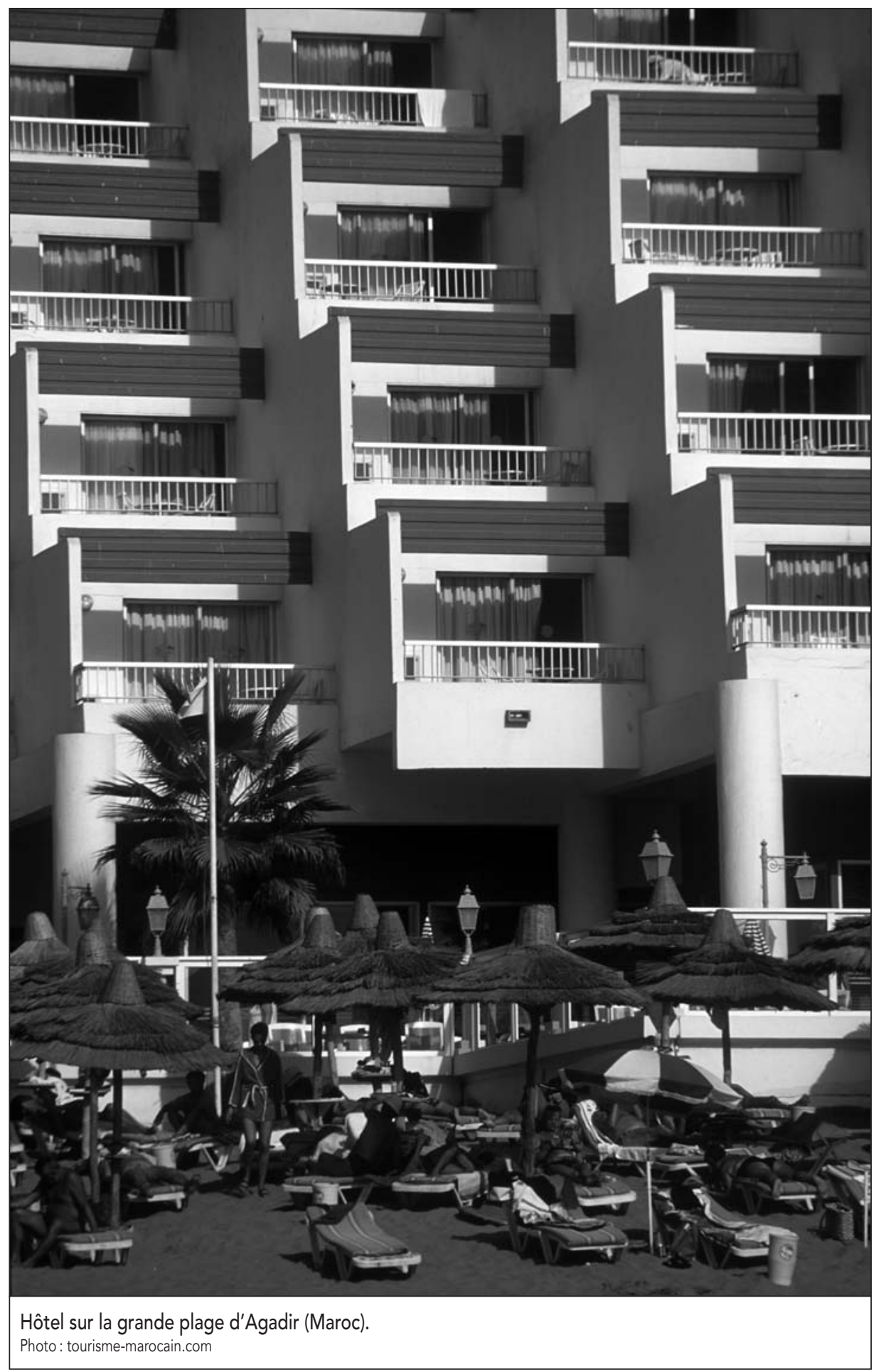

À la mode à cette époque-là, la Méditerranée s'est vue attribuée la part du lion. Est-ce cette vogue d'alors qui a séduit l'État? Ou bien y avait-il des raisons sociopolitiques? Ou encore, plus logiquement, en voyant décoller de façon rapide Agadir, sans grande assistance étatique, le gouvernement marocain, qui a toujours affiché son soutien à l'initiative privée, n'a-t-il pas éprouvé un grand soulagement en se déchargeant du fardeau de l'investissement hôtelier dans cette région pour ne plus s'occuper que de l'infrastructure, de la promotion et de la formation? Difficile à dire. Une chose est sûre: le décollage de la station d'Agadir est dû en premier lieu à l'intérêt manifesté par les classes riches et moyennes de la ville à l'égard du tourisme. Les chercheurs qui ont travaillé 
sur la région ont souligné «l'absence de l'État en tant qu'investisseur " (Péré, 1972: 3-27) et en même temps la dynamique des investisseurs de la région en rappelant (Berriane, 1979 : 254) que près de «la moitié des lits est contrôlée par le capital local ».

Comme autres paramètres, on peut citer : le site bien abrité de la ville, la plaine assez fertile du Souss, l'arrière-pays très attrayant et diversifié, une mer aux eaux tièdes, bordée par une agréable baie de sable fin, éléments qui forment un "cocktail » de riches potentialités, à tel point qu'on est autorisé à penser que lorsque le tourisme trébuche à Agadir, la faute en incombe vraisemblablement à la mauvaise gestion ou promotion !

Agadir est l'une des rares destinations littorales ouvertes toute l'année. Mieux, elle attire plus de touristes en dehors de l'été, grâce à la clémence du climat. Elle compte aussi parmi les stations touristiques marocaines réputées pour la qualité de leur animation, celle des équipements et des moyens d'accueil offerts aux touristes (salles de congrès, casinos, night-clubs en quantité et en qualité suffisantes...), comme par l'importance de ses équipements sportifs : c'est la seule ville du Royaume qui compte trois terrains de golf, en plus de 120 courts de tennis ouverts aux touristes et à la population locale, ce qui crée une ambiance d'animation, malheureusement limitée à certaines classes.

\section{La nouvelle stratégie du développement touristique au Maroc: la troisième génération de stations balnéaires est en gestation}

Sur la rive nord de la Méditerranée, le tourisme balnéaire classique (bronzage, repos, sexe...) connaît un léger recul, cependant largement compensé par l'explosion des activités ludiques, sportives, nautiques, de plaisance ou tout simplement de plaisir de vivre (festivals de musique, de théâtre, de gastronomie...). L'attrait de la mer ne faisant plus recette, seule une animation culturelle diversifiée vient renforcer l'attrait des stations balnéaires en déperdition et permettre notamment de compenser les déficits occasionnés par certaines atteintes à l'environnement. Sur la rive sud, le tourisme balnéaire s'ingénie encore à séduire par les recettes d'antan, au point de creuser notablement le décalage avec l'évolution très rapide des mentalités des pays du

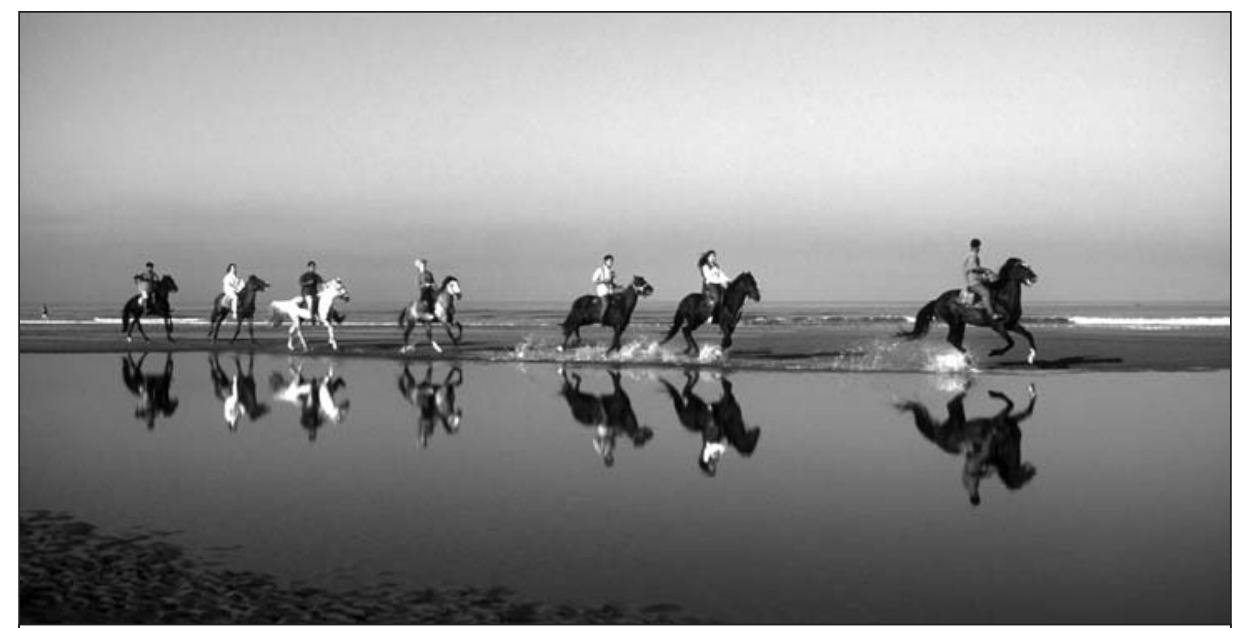

Grande plage d'Agadir (Maroc).

photo : tourisme-marocain.com

\section{Tableau 3}

Caractéristique des cinq principales nouvelles stations touristiques balnéaires du Maroc ${ }^{11}$

\begin{tabular}{l|c|c|c|c|c|c|c} 
Nom de la station & Plage blanche & $\begin{array}{c}\text { Taghazout } \\
\text { (Agadir) }\end{array}$ & $\begin{array}{c}\text { Mogador } \\
\text { (Essaouira) }\end{array}$ & $\begin{array}{c}\text { El Haouzia } \\
\text { (El Jadida) }\end{array}$ & $\begin{array}{c}\text { K. Sahel } \\
\text { (Larache) }\end{array}$ & $\begin{array}{c}\text { Saidia } \\
\text { (Oriental) }\end{array}$ \\
\hline Superficie totale & 525 ha & 868 ha & 356 ha & 475 ha & 490 ha & 600 ha \\
Capacité Générale & 26000 lits & 23000 lits & 7900 lits & 8500 lits & 15000 lits & 150 lits \\
\hline Hôtellerie classique & 28 hôtels & - & 32 hôtels & 14 hôtels & 16 hôtels & - \\
\hline
\end{tabular}

Source : Ministère du Tourisme, 2003.

Nord, fournisseurs de touristes. Le Maroc, revigoré par une nouvelle ère politique, décide de réagir en lançant le plan Azur et vise 10 millions de touristes à l'horizon 2010, dans un programme d'action ambitieux qui prévoit la réalisation de six nouvelles stations balnéaires de type «mégastations ».

Avec ce programme, qui laisse rêveur, le Maroc souhaite mobiliser toutes les énergies pour la relance du secteur touristique. Des chiffres fabuleux sont avancés et parlent de quelque 5 milliards d'euros d'investissements. L'objectif déclaré est de créer 160000 nouveaux lits et environ 600000 emplois directs et indirects pour des recettes annuelles en devises de l'ordre de 80000 milliards de dirhams en 2010. La conjoncture nationale des investissements est bonne: le Maroc trône actuellement en tête des pays africains concernant les investissements étrangers. Le début de l'opération est prometteur. II semble que la première phase de sélection des opérateurs privés, les fameux " aménageurs-développeurs ", selon l'expression consacrée, atteint à la fin de 2004 le chiffre record de 22 soumissions.
Après deux décennies de doute (1980-1990), le Maroc revient à l'idée du tourisme moteur de développement et espère une contribution du tourisme au PIB de $20 \%$ dans dix ans. Ces ambitions sont réalisables à condition d'éviter l'adage populaire: "on reprend les mêmes et on recommence ».

\section{Conclusion}

Depuis 2001, le tourisme mondial traverse une zone de turbulences et le Maroc en pâtit. À défaut d'exceller, les professionnels de ce secteur sensible s'ingénient à juguler la crise. Le tourisme, ce secteur vital pour le Royaume, continue à subir, malgré les efforts déployés par le ministère de tutelle et ses instances de soutien (augmentation de $17 \%$ des arrivées au milieu de 2004), les conséquences d'une décennie et demie (1991-2004) de chocs économiques, de conflits militaires ou d'attentats terroristes à l'échelle mondiale.

Le tourisme balnéaire, perçu au départ comme un gisement inépuisable (mer, soleil, plage), a pâti de certaines erreurs d'aména- 
gement, dues à l'inexpérience et à une gestion confuse par le secteur public, qui ont sérieusement altéré les meilleures plages et côtes du Nord, finissant par discréditer l'image de marque d'une destination qui a fait un certain temps la fortune et la gloire des villes balnéaires méditerranéennes. Ajoutons à cela la crise énergétique de 1973, qui a éliminé l'avantage de la proximité du Maroc des grands foyers émetteurs de touristes (Espagne, France...), en rendant le voyage individuel en voiture plus onéreux que les voyages à forfait en avion... En conséquence, l'État s'était trouvé dans l'obligation de prendre en charge la promotion et même la gestion des nouvelles unités balnéaires, avant de les céder à la gestion étrangère et plus tard au secteur privé.

Les pouvoirs publics vont s'attacher, de façon rétroactive, à consolider les acquis, à corriger les erreurs du passé et, en même temps, à assurer le bon fonctionnement et la continuité des secteurs et des branches d'activités qui ne sont pas déficitaires, sans jamais se départir de la ligne politique tracée au début des années 1960. Une façon habile qui consiste à rectifier sans cesse le tir sans se désavouer (ligne idéologique oblige). Mais quel gâchis! Ces opérations avortées (cas du balnéaire nord) ont des conséquences négatives sur la région et le secteur:

- Sur le plan local, elles ont occupé les meilleurs sites au détriment d'autres vocations (pêche, industrie, urbanisme...) et ont fini par livrer les terrains acquis à des prix raisonnables ou par expropriation à une spéculation foncière contraire à l'esprit d'utilité publique pour lequel ils ont été acquis.

- À l'échelle régionale, elles ont absorbé d'importants capitaux publics, sous prétexte d'aide au secteur productif prioritaire au détriment, de toute évidence, des secteurs sociaux. Ces derniers ont dû subir un second laminage avec la mise en place du Programme d'ajustement structurel.

- Au niveau sectoriel, ces revers ont terni l'image d'un secteur naissant porteur d'espoirs. Heureusement que le Sud atlantique et les villes impériales, des régions où l'État n'a rien fait de remarquable, ont pu résister à la crise et limiter les dégâts.

Sur cette même lancée d'interrogations, il va falloir se demander quel est le prix social, économique et surtout environnemental des erreurs d'appréciation et des mauvaises gestions d'un secteur porteur, mais usé avant même d'avoir fait ses preuves. Question très délicate, surtout s'il faut formuler des arguments chiffrés.

Enfin, l'espoir est grand que les nouvelles stations tirent profit des leçons du passé. Le grand changement survenu au Maroc en 1999 permet de caresser cette espérance.

Mimoun Hillali est enseignant-chercheur à I'Institut supérieur international du tourisme de Tanger (Maroc); ses travaux de recherche s'intéressent aux divers aspects du tourisme et ses relations avec l'espace, l'environnement et la culture.

\section{Notes}

1 En février 1964, à la demande du Maroc, la Banque mondiale envoie une équipe d'experts afin «d'évaluer les potentialités de développement de l'économie du pays [...]" (d'après la BIRD. 1966).

2 L'Algérie indépendante (1962) a opté pour le socialisme et le parti unique. Elle avait, à l'époque, des admirateurs dans la jeunesse et la classe politiques marocaines.

3 La décision vers 1777 de Sidi Mohamed Ben Abdellah de concentrer tous les consuls étrangers à Tanger, jusque-là dispersés aux quatre coins du royaume, a fait de Tanger un joyau du cosmopolitisme et du tourisme et a vu naître le premier hôtel moderne du Maroc vers 1852.

4 Fêtes foraines, manifestations religieuses, cérémonies populaires, Moussems...

5 Les bêtes et les marchandises occupaient le rez-de-chaussée et les marchands ou voyageurs les étages supérieurs (en général deux). De nos jours, l'hôtel s'appelle en arabe Al Foundouk, mais il désigne aussi dans la campagne marocaine l'enclos réservé aux bêtes de somme à proximité d'un Souk.

6 C'est le Maréchal Lyautey qui, convaincu du profit que pourrait retirer le protectorat du tourisme au Maroc, posa en 1921 la première pierre d'un hôtel de grand luxe à Marrakech...

7 Discours prononcé par feu Hassan II à l'ouverture de la session du Conseil supérieur de la promotion nationale et du plan, le 21 décembre 1964.

8 Zone d'aménagement touristique prioritaire.

9 Le séisme du 22 février 1960 a fait près de 20000 morts et causé des dégâts matériels importants. Sur le plan touristique, la ville repart à zéro.

10 La Sonaba est déclarée d'utilité publique par le Dahir portant loi n 1-76-393 du 21 juin 1976.

11 Les données chiffrées concernant ces stations changent fréquemment en fonction des aspirations ou des capacités des éventuels investisseurs aménageurs.

\section{Bibliographie}

Bahraoui-Buret, J., M.N. Bargach, et M.L. Kaddour (1983), Le gisement solaire marocain, Rabat, Société Marocaine des Éditeurs Réunis (SMER).

Berriane, Mohamed (1979), "Recherches géographiques et cartographiques sur les espaces touristiques au Maroc ", thèse de troisième cycle, Université François Rabelais, Tours, France.

Besancenot, Jean-Pierre (1989), Climat et tourisme, Paris, Édition Masson, Collection géographie.

BIRD (Banque internationale pour la reconstruction et le développement) (1966), The Economic Development of Morocco, The Johns Hopkins Press, Baltimore, USA.

Grande encyclopédie du Maroc (La) (1987), Volume Géographie humaine, Italie GEI, Cremon.

Miossec, Jean-Marie (1993), «Tourismes dans le monde arabe : Méthodes et concepts ", dans Centres d'études et de recherches URBAMA (Urbanisation du Monde Arabe), Recherches urbaines dans le monde arabo-musulman, Université de Tours, France, fascicule de recherches $n^{\circ} 24$, p. 97-114.

Hillali, Mimoun (1985), « Le développement du tourisme sur la côte méditerranéenne du Maroc: potentiel et action gouvernementale ", thèse de troisième cycle en urbanisme, Université d'Aix-Marseille III. IAR, Aix-en-Provence, $350 \mathrm{p}$.

Hillali, Mimoun (2000), "La politique touristique marocaine ", thèse d'État, Université de Liège, Belgique, faculté des sciences, Institut de géographie.

Hillali, Mimoun (2003), Le tourisme international vu du Sud: essai sur la problématique du tourisme dans les pays en développement, Ste-Foy, Les Presses de l'Université du Québec, collection «Tourisme».

Ministère du Tourisme (1970-2002), Annuaires des statistiques du tourisme.

Ministère du Tourisme (2003), Le secteur touristique (statistiques 2001-2003), Direction de la planification et de la coordination de la promotion, Rabat, Maroc.

Péré, Michèle (1972), "Quelques aspects du tourisme au Maroc à travers l'exemple d'Agadir ", Revue de géographie du Maroc, $\mathrm{n}^{\circ} 22$, p. 3-27.

Salmi, Jamil (1989), Planification sans développement: Évaluation de l'expérience marocaine de planification 1960-1979, Casablanca, Les Éditions Maghrébines.

Sebbar, Hassan (1976), «Tourisme et développement, le cas du Maroc", Bulletin économique et social du Maroc, $\mathrm{n}^{\circ} 127$.

Stafford, J., B. Sarrasin, et C.É. Bélanger (1996), Développement et tourisme au Maroc, Montréal, Harmattan. 